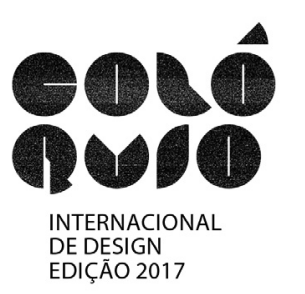

\title{
Biomimética e Design: um estudo sobre a potencialização da criatividade para métodos de desenvolvimento de produtos inspirados na natureza
}

\author{
Daniel de Souza Gamarano; \\ Victória Carolina Pinheiro Lopes Dias; \\ Thaís Falabella Ricaldoni
}

resumo:

O presente artigo visa discutir as metodologias de desenvolvimento de produtos que tem como base os processos de Biomimética, a partir da avaliação dos processos de criatividade humana. Inicialmente buscou-se contextualizar sobre a criatividade e seus mecanismos, abordando sobre as dimensões da criatividade e inteligência, e a sua relação com os pensamentos divergente e convergente. Posteriormente, são abordados aspectos ligados aos métodos de Design vinculados aos processos naturais, aqui estudados, a partir dos princípios da Biomimética. Este campo de conhecimento apresenta uma investigação sistemática das soluções orgânicas e estruturais aplicadas pela natureza aos seus elementos, visando colher dados para a solução de problemas técnicos de formas, estruturas ou objetos para o uso cotidiano humano. Autores do domínio da Psicologia, como Guilford (1977) e Cropley (2001), e da Biomimética, como Beynus (1997), foram estudados e suas teorias utilizadas como fundamentação para as reflexões que se seguiram sobre a análise de experiência real sobre a aplicação de princípios biomiméticos em um ambiente de ensino de Design no Brasil, da Universidade Federal de Pernambuco, no laboratório de BioDesign (UFPE). O método estudado foi o de Analogia, no qual os princípios naturais são interpretados, desenvolvidos e transformados em possíveis soluções que podem ser utilizadas no fomento de ideias para novos produtos. Foram verificados que essa abordagem tem associação dos parâmetros da estruturação do pensamento divergente e convergente. Essa estrutura de pensamento tende a potencializar as dimensões da criatividade. Como apontamento futuro, relacionar mecanismos da estruturação do pensamento criativo com os princípios da Biomimética tende a promover a geração de novos métodos que possam auxiliar o desenvolvimento e um maior aproveitamento da gama de informações que podem ser retirados de sistemas naturais complexos.

palavras-chave:

Design; Biomimética; Criatividade; Ensino; Desenvolvimento de Produtos. 


\section{Contextualização sobre a criatividade e seus mecanismos}

A criatividade é um elemento primordial para diversas áreas do conhecimento, mesmo as mais tradicionais vêm incorporando o termo. Muitos estudos abordam o tema da criatividade, como teses, dissertações acadêmicas e artigos. Para Parolin (2008) a criatividade é um desejo básico do ser humano, atuando no anseio da geração de ideias, sendo a inovação o processo pelo qual as ideias criativas se transformam em processo, métodos, produtos ou serviços.

A curiosidade é uma força que move criatividade, através dela somos motivados a investigar através de uma série de processos criativos programados ou não. "O exercício da curiosidade convoca a imaginação, a intuição, as emoções, a capacidade de conjecturar, de comparar, na busca pela perfilização do objeto ou do achado de sua razão de ser" (Freire, 1996, p.98).

Para definir a criatividade, White (2015) afirma ser necessário acabar com a crença de que ela é algo raro e que poucas pessoas a possuem. E ainda que esteja mais próxima de uma determinada faixa etária, gênero ou profissão. A criatividade é uma função particular da mente, todas as pessoas têm e podem desenvolvê-la através da prática de alguns métodos. A autora ainda ressalta que existem quatro dimensões da criatividade: fluidez, flexibilidade, originalidade e a elaboração. Essas dimensões interagem entre si a partir da fluidez, que é a habilidade de gerar um grande número de ideias num curto espaço de tempo, estas têm que ser flexíveis e originais, e possibilitar a elaboração, que só é possível ser feira através de ideias válidas e de conteúdo utilizável.

A interação entre as quatro dimensões pode ser ampliada, se os indivíduos utilizarem de forma eficiente, as duas formas de pensamento, convergente e divergente. $\mathrm{O}$ conceito de pensamento convergente e divergente foi introduzido pelo psicólogo americano Paul Guilford (1977), que observou haver dois tipos de resposta a um problema, e chamando-as de produção convergente e divergente. Guilford (1977) define o pensamento convergente (vertical) como sendo lógico, analítico, linear, verbal e sequencial, seu desenvolvimento está ligado ao hemisfério esquerdo do cérebro, já o pensamento divergente (lateral) surge no hemisfério direito do cérebro é visual, emotivo, e sua produção mental não é linear. Alternar esses dois pensamentos de forma eficiente pode oportunizar aos indivíduos a construção de uma síntese de levantamento para estruturar uma nova realidade, a este processo mental chamamos de criatividade. A figura 1 representa uma síntese dos pensamentos convergente e divergente.

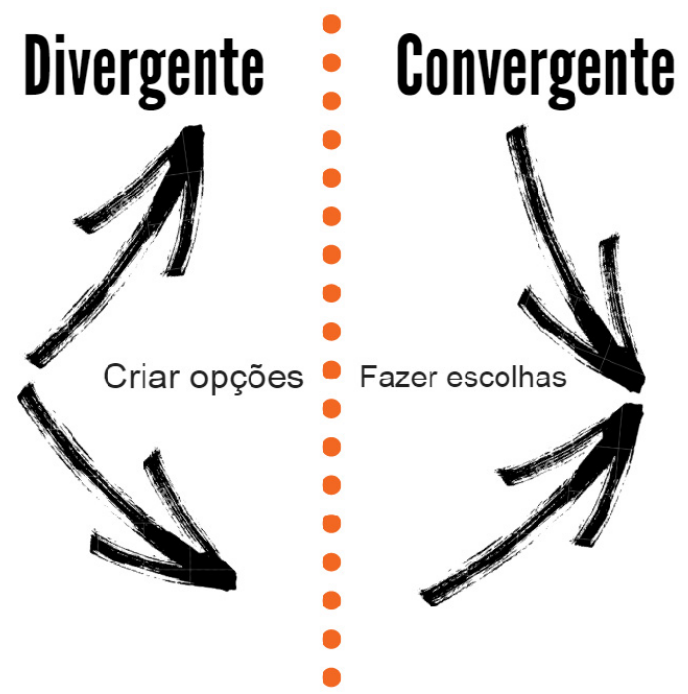

Fonte: Elaborado pelos autores 
Cropley (2001) faz uma abordagem quantitativa a respeito dos vários níveis de criatividade conhecidos, indo do nível simples, ligado a ações cotidianas, ao nível sublime, que abrange ações complexas.

O autor ressalta que as primeiras pesquisas realizadas nas décadas de 1950 e 1960 consideram a criatividade e a inteligência como campos que deveriam ser analisados separadamente, mas o fato é que ambos traçam caminhos semelhantes. Por isso existem limites comuns: como as habilidades cognitivas para a definição de problemas, e codificação seletiva.

Mas o fato inegável é que, para se ter criatividade, há a junção dos dois termos: a inteligência e a própria criatividade, mas para um indivíduo inteligente ser criativo ele deve ter essa performance em criar novos contextos. A tabela I apresenta as diferenças entre criatividade e inteligência.

Tabela I: Domínios psicológicos da inteligência e da criatividade

Domínio Psicológico

\begin{tabular}{cll}
\hline \multicolumn{1}{c}{ Função } & $\begin{array}{l}\text { Conhecimento feito a partir dos } \\
\text { fatos já conhecidos } \\
\text { (produção ortodoxa) }\end{array}$ & $\begin{array}{l}\text { Desenvolvimento de novos } \\
\text { caminhos } \\
\text { Produção de novidade }\end{array}$ \\
\hline Habilidades inerentes & $\begin{array}{l}\text { Fazer pensar } \\
\text { Solução de problemas }\end{array}$ & $\begin{array}{l}\text { Imaginação } \\
\text { Solução de problemas }\end{array}$ \\
\hline $\begin{array}{c}\text { Habilidades que podem ser } \\
\text { desenvolvidas }\end{array}$ & $\begin{array}{l}\text { Pensamento Convergente } \\
\text { Processo de pensamento }\end{array}$ & $\begin{array}{l}\text { Pensamento divergente } \\
\text { Pensamento crítico }\end{array}$ \\
& Pensar o conhecido & Inventividade \\
& Reconhecimento do familiar & Link com domínios diferentes \\
Ramificações
\end{tabular}

Fonte: Adaptado de Cropley (2001)

\section{Design e processos naturais: a abordagem biomiética}

Ao longo da historiografia do Design, seus métodos necessitaram ser revistos e os designers sempre foram desafiados a mudar a sua perspectiva diante dos contextos que se configuravam. O design notadamente é uma área do conhecimento que utiliza processos criativos em seus métodos, sendo ele uma disciplina que lida constantemente com o desenvolvimento ou o aprimoramento de sistemas cada vez mais complexos, como expõe o autor Moraes (2013), ao afirmar que o desafio dos designers na atualidade é atuar em cenários cada vez mais mutantes e complexos. Sendo assim, novos métodos para potencializar a criatividade e sustentar a inovação precisam ser adaptados e desenvolvidos.

Para a autora Soares (2008):

A natureza foi, é, e será uma fonte infinita de inspiração criativa para a humanidade. Os sistemas biológicos que residem na natureza são caracterizados pela sua complexidade, sensibilidade e flexibilidade, pela sua capacidade de adaptar-se a ambientes em mudança, e pelo seu elevado grau de fiabilidade. (Moraes, 2008, p.15)

Pode-se considerar a natureza um elemento útil para o desenvolvimento da criatividade, neste contexto a Biomimética se apresenta como uma fonte para o incremento criativos dos métodos de design. Para Guimarães (2011), a natureza pode ser comparada a um grande designer, o qual projeta muitos subsistemas. $\mathrm{O}$ autor afirma serem três os princípios básicos que guiam os projetos da natureza: otimização, ciclo fechado e interdependência. Não há desperdício na natureza, sempre são encontradas formas que aperfeiçoem os materiais e a energia. Da mesma forma, tudo acontece em ciclos: das marés, do sol, da lua, as estações. Ademais, cada acontecimento gera suas consequências, que darão 
fomento a outros acontecimentos. A Biomimética é uma área da ciência que estuda as estruturas biológicas e suas funções, procurando compreender a natureza, para tirar dela algum ensinamento, ou conceito básico. A palavra Biomimética é de origem grega, proveniente das palavras bíos e mímesis, significando 'a imitação da vida'. Além desse termo, tem-se a Biônica e a Biomecânica, compreendidas como as ciências que estudam determinados processos biológicos dos seres vivos (mecanismos mecânicos e eletrônicos) a fim de aplicar processos análogos na área técnica e na indústria (GUIMARÃES, 2011).

De maneira geral, todos esses termos apontam para a investigação sistemática das soluções orgânicas e estruturais aplicadas pela natureza aos seus elementos, visando colher dados para a solução de problemas técnicos de formas, estruturas ou objetos para o uso cotidiano humano. Há algum tempo, a Biomimética tem sido uma ferramenta para designers, que possibilita encontrar soluções projetuais a partir de elementos da natureza. Sua importância é tanta, que diversos centros universitários já possuem disciplinas e estudos na área, se destacando países como Suíça, Itália, Japão e Estados Unidos.

O processo criativo de um projetista não conta somente com a inspiração, mas também com métodos e ferramentas que permitam a manifestação da criatividade. Esta pode surgir de inúmeros ambientes, inclusive, da própria natureza (DETANICO; TEIXEIRA; SILVA, 2010).

Janine Benyus foi uma das primeiras estudiosas a respeito do tema, lançando o livro Biomimicry - Innovation Inspired by nature que define os conceitos básicos dessa abordagem. São eles:

a) Natureza como modelo: Estudar os modelos da natureza e imitá-los ou usá-los como inspiração, com o intuito de resolver os problemas humanos;

b) Natureza como uma medida: Usar o padrão ecológico para julgar a relevância e a validade das nossas inovações. Após bilhões de anos de evolução, a natureza aprendeu o que funciona, o que é mais apropriado e o que perdura;

c) Natureza como um mentor: Nova forma de observar e avaliar a natureza. Preocupar-se não no que podemos extrair do mundo natural, mas no que podemos aprender com ele (BEYNUS, 1997). A tabela II apresenta os princípios de biomimetismo defendido por Beynus (1997):

Tabela II: Princípios de Biomimetismo

\begin{tabular}{c}
\hline JANINE BENYUS (1997) \\
\hline Uso de resíduos como recurso \\
\hline Diversificação e cooperação para usar completamente o habitat \\
\hline Obter energia de forma eficiente \\
\hline Otimizar em vez de maximizar \\
\hline Desmaterialização \\
\hline Minimizar a poluição e o uso de recursos \\
\hline Partilhar a informação \\
\hline Consumir localmente
\end{tabular}

Fonte: adaptado de Beynus (1997) 
O Biomimicry Institute é um grupo de pesquisa que visa divulgar metodologias que permitam a realização de projetos com Biomimética. Eles possuem um portal, o Ask Nature, que avalia diversos aspectos funcionais da natureza, além de cases utilizados no design de produto, sendo uma importante fonte de pesquisa. O método de desenvolvimento de projetos com abordagem em Biomimética consiste em três etapas fundamentais: como explorar um princípio natural, como descobrir um conceito de projeto para a estrutura e como criar este conceito. A figura 2 apresenta uma explicação gráfica sobre o processo

Figura 2: Síntese do processo Biomimético

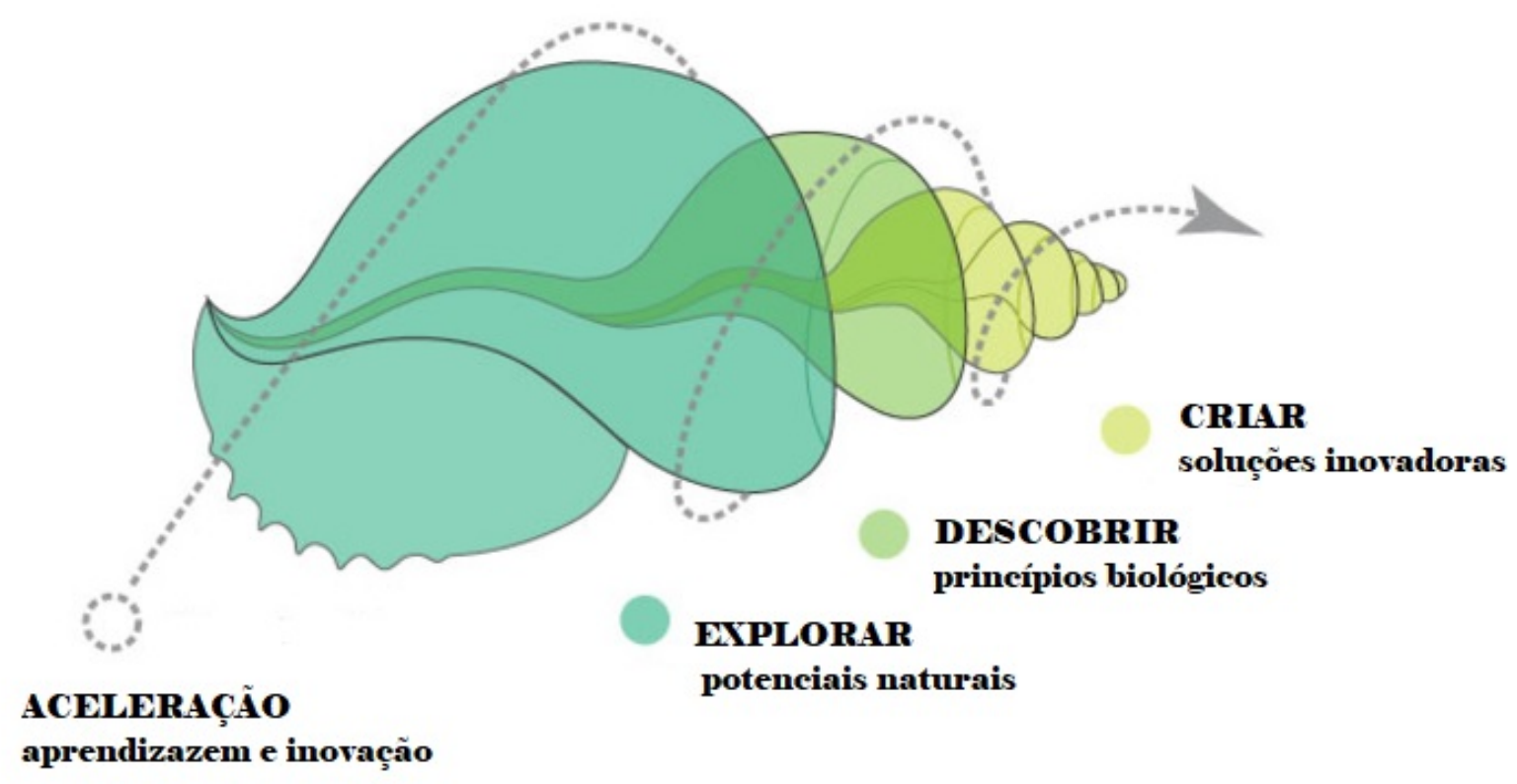

Fonte: Adaptado de Ask Nature (2017)

Koehler (2014), pesquisadora do instituto, apresenta uma metodologia que une as etapas da Biomimética com o pensamento convergente e divergente no processo, focando a cada etapa, do processo, em soluções que ampliam as discussões para a posterior convergência de ideias e criação de soluções, como apresentado na figura 3. 
Figura 3: Espiral da Biomimética alinhada ao pensamento divergente e convergente

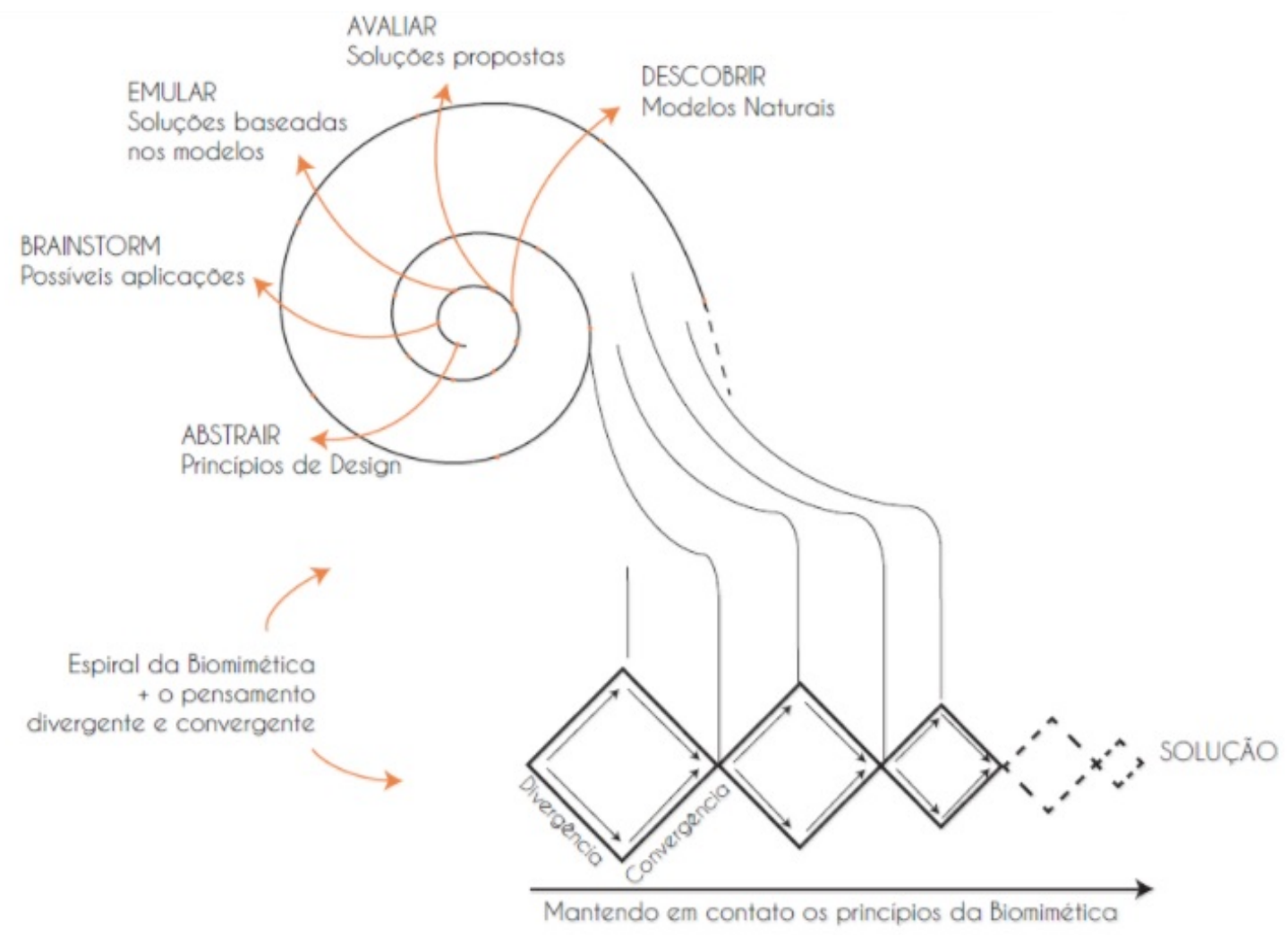

Fonte: Adaptado de Koehler (2014).

\section{Reflexões sobre a aplicação da pesquisa no ambiente de ensino em Design a partir de princípios biomiméticos}

A seguir utilizaremos como base um experimento realizado no ambiente de ensino em design, com a proposta de estimular a criatividade dos discentes, este foi realizado na Universidade Federal de Pernambuco, Brasil. Em um contexto acadêmico, se entende a Biomimética como uma ciência empírica que utiliza exercícios de maneira experimental, fazendo analogia com as estruturas naturais. $\mathrm{O}$ intuito do projeto foi o de desenvolvimento e estímulo da capacidade criativa dos alunos, tendo como base a pesquisa de formas, cores e texturas naturais, os quais pudessem ser potencialmente interpretados e utilizados em desenvolvimento de projetos de design.

Uma das abordagens acadêmica é através da Analogia, ou seja, a busca soluções a partir da tradução das características estruturais e formais, que são transpostas para os produtos. Segundo os autores (SOARES et al, 2017), é possível analisar o estudo da Analogia por meio da análise de três campos: a Formação, a Pesquisa e o Projetual.

O campo da Formação se refere ao âmbito do ensino, ao considerar a didática e os processos, sempre avaliando os aspectos naturais. É realizada mediante o estímulo de experimentação e abstração, com o intuito de fazer um panorama da filosofia por detrás de um projeto biomimético.

O campo da Pesquisa compreende o ato de desenvolver estudos capazes de gerar resultados às indagações propostas primeiramente. Deve considerar uma equipe interdisciplinar, de maneira a influenciar o diálogo e a construção de parâmetros em conjunto. A Pesquisa pode ser tanto de base, ou seja, de caráter bibliográfico, teórico; quanto aplicada, através da confecção de modelos e protótipos que favoreçam a análise.

O campo Projetual refere-se aos princípios Biônicos, que, por terem alto grau de complexidade, são divididos em três possibilidades de intervenção, como linguagem, instrumento e hipótese de projeto.

O método desenvolvido pelo laboratório de BioDesign (UFPE/BRA) consistia em utilizar a Analogia de organismos vivos, para serem aplicados na resolução de algum problema de projeto. Para 
tanto, eles utilizaram ferramentas como: a representação fotográfica, descrição verbal e esquematização por desenho e modelos. Segundo Soares et al (2017), a representação fotográfica deve ter o maior número de detalhes quanto for possível, a descrição verbal se concentra na busca de informações sobre o elemento natural analisado, como detalhes técnicos e históricos, bem como dados relativos aos detalhes da fotografia. A esquematização é feita através de desenhos, os primeiro devem ser desenhos de observação, posteriormente formas são abstraídas deles, com o critério de manter a coerência com elemento natural analisado. O processo é finalizado com a confecção de um modelo tridimensional, podendo este ser também virtual. A figura 4 abaixo exemplifica o esquema do método utilizado pelo autor.

Figura 4: Esquema do método de Analogia desenvolvido.

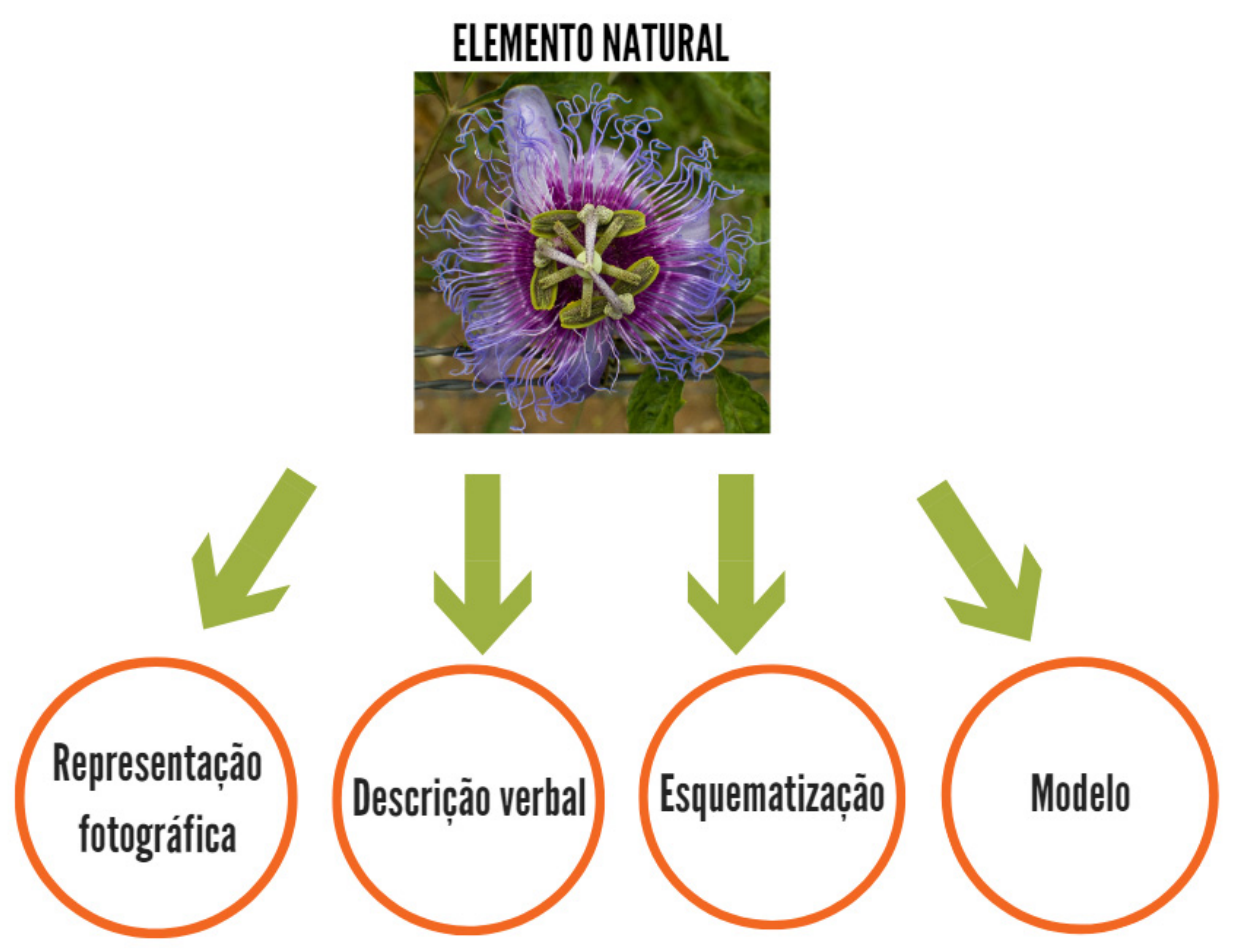

Fonte: Adaptado de SOARES et al, 2017

Seguindo o método desenvolvido pelo laboratório, os discentes desenvolveram seus projetos, como os estudos análogos a seguir. O primeiro projeto foi realizado a partir da síntese geométrica das folhas do abacaxi. O segundo é um estudo feito através da análise óssea da articulação do ombro e do cotovelo humano. A amplitude do movimento feito por estes membros foram adaptados para o desenvolvimento de um protótipo articulado, como apresentado na figura 5. 
Figura 5: Resultados do estudo de Analogia.
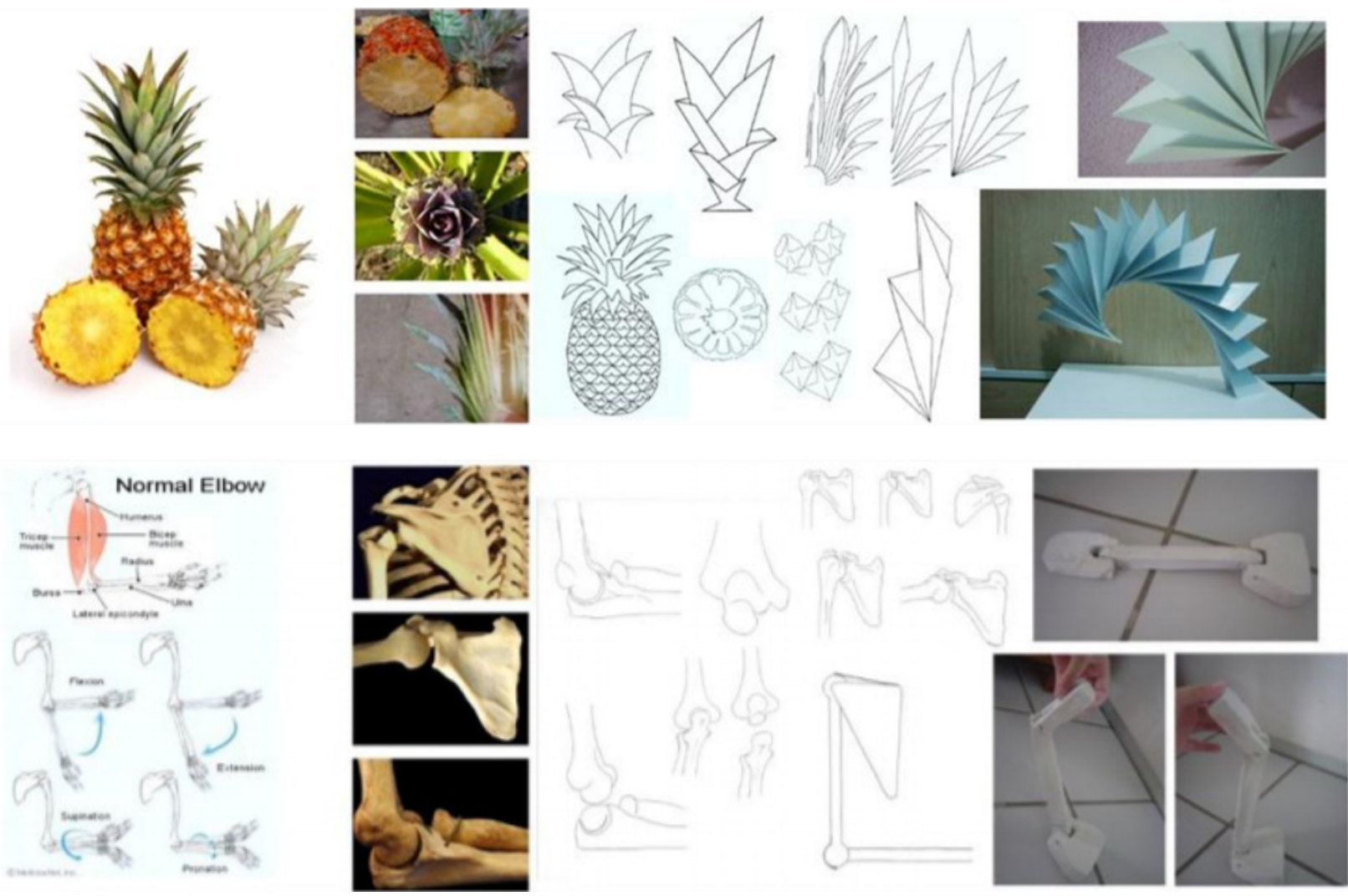

Fonte: Fonte: SOARES et al, 2017

\section{Considerações finais}

Diante do exposto, pode-se concluir que há estudos pertinentes utilizando a Biomimética em projetos de Design, porém a muito a ser explorado. Como apresentado pelos resultados gerados pela experiência dos pesquisadores do departamento de Design da Universidade Federal de Pernambuco, é possível estruturar métodos biomiméticos a partir de reflexões em sistemas e fenômenos mais complexos, como proposto por Beynus (1997).

A estruturação do método de Analogia foi realizada em parâmetros associados à convergência e divergência de pensamentos, presenciadas em todas as etapas do projeto, desde a etapa de ideação, mais ligadas ao pensamento divergente, até a definição da melhor solução gerada, relacionada com o pensamento convergente. Destaca-se, ainda, que a pesquisa e levantamento bibliográfico prévio, bem como os primeiros protótipos são realizados mediante a uma construção de repertório e pode permitir o desenvolvimento de pensamentos criativos para possíveis soluções de projeto de produto.

Como apontamento futuro, relacionar mecanismos da estruturação do pensamento criativo com os princípios da Biomimética tende a promover a geração de novos métodos que possam auxiliar o desenvolvimento e um maior aproveitamento da gama de informações que podem ser retirados de sistemas naturais complexos. 
Biomimetics and Design: a study on the potentialization of creativity for
methods of development of products inspired by nature Abstract:

The present article aims to discuss the methodologies of product development based on the biomimetic processes, based on the evaluation of human creativity processes. Initially we sought to contextualize about creativity and its mechanisms, addressing the dimensions of creativity and intelligence, and its relation to divergent and convergent thoughts. Subsequently, aspects related to the Design methods related to the natural processes, studied here, are approached from the principles of Biomimetics. This field of knowledge presents a systematic investigation of the organic and structural solutions applied by nature to its elements, aiming to gather data for the solution of technical problems of forms, structures or objects for daily human use. Authors of the field of Psychology, such as Guilford (1977) and Cropley (2001), and Biomimetics, like Beynus (1997), were studied and their theories used as a basis for the following reflections on the analysis of real experience on the application Of biomimetic principles in a teaching environment of Design in Brazil, of the Federal University of Pernambuco, in Bio Design laboratory (UFPE). The method studied was that of Analogy, in which natural principles are interpreted, developed and transformed into possible solutions that can be used in the promotion of ideas for new products. It was verified that this approach has an association of the parameters of the structuring of divergent and convergent thinking. This structure of thought tends to potentiate the dimensions of creativity. As a future point of reference, linking the mechanisms of the structuring of creative thinking with the principles of Biomimetics tend to promote the generation of new methods that can aid development and a greater use of the range of information that can be extracted from complex natural systems.

Keywords: Design; Biomimetics; Creativity; Teaching; Product Development.

\section{Referências bibliográficas}

ASK NATURE, 2017. Site oficial. Disponível em http://www.asknature.org/strategy/b4d8f858db37c80adfbd6543b4ef526. Acesso 20/05/2017

BEYNUS, J. Biomimicry: Innovation Inspired by Nature. Editora William Morrow \& Co., Califórnia, 1997.

CROPLEY, A. Creativity in education and learning - a guide for teachers and educators Routledge Taylor e Francis Group, London / New York, 2001.

DE MORAES, D. Metaprojeto o design do design. São Paulo: Blucher, 2011

FREIRE; P. Pedagogia da autonomia. Saberes necessários à prática educativa. Paz e Terra. Rio de Janeiro: 1996

DETANICO, F.B.; TEIXEIRA, F.G.; SILVA; T.K. A biomimética como método criativo para o projeto de produto. 2010 Pg Design, Design e Tecnologia, UFRGS.

GUILFORD; P. J. La naturaleza de la inteligencia humana, Paidós. Buenos Aires: 1977

GUIMARÃES, M. Na natureza tudo se cria (página 11 a 15). ABC DESIGN. Edição trimestral Julho/Agosto/Setembro de 2011.

KOEHLER, S. Aspiring to improve the world by crafting a career in sustainable Design Part 1: A new way of thinking / Part 2: Putting Theory into Practice / Part 3 Learning from Nature. (2014). Disponível

em

http://www.core77.com/blog/sustainable_design/aspiring_to_improve_the_world_by_crafting_a_caree r_in_sustainable_design_part_3_learning_from_nature_26554.asp. Acesso 20 set. 2014. 
PAROLIN; S.R.H. As características das organizações para criatividade em organizações inovativas. Tese. Usp disponível em : http://www.teses/disponiveis/12/.../tese_SRHPParolin.pdf. acesso em 25 de junho de 2017

SOARES, M. A. R. Biomimetismo e Ecodesign: Desenvolvimento de uma ferramenta criativa de apoio ao design de produtos sustentáveis.Dissertação apresentada na Faculdade de Ciências e Tecnologia da Universidade Nova de Lisboa para a obtenção do grau de mestre em Engenharia do Ambiente. 2008.

SOARES, T.L.F.S; ARRUDA, A.J.V; MELLO, B.L.A.; OLIVEIRA, B.A.R.M.Análises sobre uma Metodologia Biomimética Aplicada em Curso de Design no Brasil DISEÑOCONCIENCIA. IX Congreso Internacional de Diseño de La Habana, Cuba 2017.

WHITE; O. M. Teoria e prática da pesquisa aplicada. Elsevier. São Paulo: 2011 